\title{
Características físicas e nutricionais de pequis oriundos dos estados de Tocantins, Goiás e Minas Gerais
}

\author{
Physical and nutritional characteristics of pequi fruits from Tocantins, \\ Goiás and Minas Gerais States
}

\section{Autores | Authors}

Aline Medeiros ALVES

Universidade Federal de Goiás (UFG) Faculdade de Nutrição Laboratório de Nutrição Experimental Goiânia/GO - Brasil e-mail: amedeiros87@gmail.com

Daniela Canuto FERNANDES

Amanda Goulart de Oliveira SOUSA

Pontíficia Universidade Católica de Goiás (PUC Goiás)

Departamento de Enfermagem, Nutrição e Fisioterapia

Goiânia/GO - Brasil

e-mail:daninutufg@gmail.com goulart.amanda@gmail.com

Ronaldo Veloso NAVES

Universidade Federal de Goiás (UFG) Escola de Agronomia Departamento de Horticultura Goiânia/GO - Brasil e-mail: ronaldo@ufg.br

\section{\ Maria Margareth Veloso NAVES}

Universidade Federal de Goiás (UFG) Faculdade de Nutrição Laboratório de Nutrição Experimental Rua 227, quadra 68, Setor Leste Universi-

CEP. $74605-0$ tário

Goiânia/GO - Brasil e-mail:mmvnaves@gmail.com

$\triangle$ Autor Correspondente / Corresponding Author

Recebido: Dez. 10, 2013 Aprovado: Set. 08, 2014
Resumo

O pequi é um fruto nativo do Cerrado que apresenta uma grande diversidade em suas características físicas e químicas. Estudos com frutos oriundos de diferentes ambientes de Cerrado são escassos na literatura. O objetivo deste estudo foi analisar as características físicas e nutricionais de frutos de pequizeiro oriundos dos estados de Tocantins (TO), Goiás (GO) e Minas Gerais (MG). Realizou-se a caracterização física de 30 frutos de pequi selecionados aleatoriamente, por estado, e determinou-se a composição química de sua polpa. Os frutos de pequi oriundos de MG apresentaram valores elevados para características físicas de importância comercial, como massa dos frutos, massa da polpa e rendimento de semente (amêndoa). Entretanto, os frutos oriundos de GO apresentaram alto rendimento de polpa. Foi observado conteúdo reduzido de lipídios nos frutos de TO $\left(8 \mathrm{~g} .100 \mathrm{~g} \mathrm{~g}^{-1}\right)$, ao contrário dos frutos provenientes de GO e MG (24 e $26 \mathrm{~g} .100 \mathrm{~g}^{-1}$, respectivamente). Em relação ao conteúdo de minerais, a polpa de pequi dos três estados constitui fonte de ferro, zinco, fósforo e magnésio, e a polpa de pequi oriundo de TO apresentou teor considerável de cálcio (107 mg.100 g $\mathrm{g}^{-1}$ ). Conclui-se que a região de origem influencia nas características físicas e químicas dos frutos, sobretudo no rendimento de polpa, nos teores de umidade e lipídios, na densidade energética e no conteúdo de cálcio da polpa de pequi. Esses resultados indicam a necessidade de caracterização da região de origem e das espécies de pequizeiro para a comercialização dos frutos e utilização da polpa como matéria-prima nutritiva em diferentes sistemas alimentares.

Palavras-chave: Caryocar brasiliense; Cerrado; Frutiferas nativas; Composição química.

\section{Summary}

Pequi is a native fruit from the Brazilian Cerrado with a great diversity in its physical and chemical characteristics. Studies with fruits from different environments of the Cerrado are scarce. The objective of this study was to analyze, physically and nutritionally, pequi fruits from Tocantins (TO), Goiás (GO) and Minas Gerais (MG) States. The physical analysis of 30 pequi fruits, randomly selected by region, was carried out, and the chemical composition of their pulps was determined. The pequi fruits from MG showed high values of commercially important physical characteristics, as fruit mass, pulp mass and seed (almond) yield. However, fruits from GO showed high pulp yield. Low lipid contents $\left(8 \mathrm{~g} .100 \mathrm{~g}^{-1}\right)$ were observed in the fruits from TO, on the contrary of the fruits from $\mathrm{GO}$ and $M G$ (24 and $26 \mathrm{~g} \cdot 100 \mathrm{~g}^{-1}$, respectively). Regarding the mineral content, the pequi pulp from the three regions is a source of iron, zinc, phosphorus and magnesium, and the pequi pulp from TO showed considerable contents of calcium (107 mg. $100 \mathrm{~g}^{-1}$ ). The native region of the fruits influences their physical and chemical characteristics, mainly the pulp yield, moisture and lipid contents, energy density and calcium amount of the pequi pulp. These results indicate the necessity of characterization of the native region and the pequi species for commercial use of the fruits and for utilization of the pulp as a nutritious raw material, in different food systems.

Key words: Caryocar brasiliense; Cerrado; Native fruits; Chemical composition. 


\section{Introdução}

O bioma Cerrado apresenta uma ampla diversidade genética intra e interespecífica em sua flora, que inclui diversas espécies frutíferas com grande potencial de utilização agrícola e tecnológica. No entanto, a exploração da maioria de suas plantas nativas está ocorrendo de maneira predatória e não sustentável, ameaçando a preservação das espécies, o que torna o estudo delas de grande importância para manejo adequado e melhor aproveitamento na alimentação humana (BATLLEBAYER et al., 2010; NOVAES et al., 2013).

O pequizeiro (Caryocar brasiliense Camb.) é uma árvore frondosa, nativa do Cerrado, que atinge de oito a dez metros de altura e frutifica de setembro a março (SANTANA e NAVES, 2003). Seu fruto, o pequi, é constituído por exocarpo de coloração marromesverdeada, mesocarpo externo formado por uma polpa branca e mesocarpo interno, porção comestível do fruto, de coloração amarelo-claro a alaranjado escuro. O endocarpo espinhoso do pequi protege a semente comestível, que é revestida por um tegumento fino e marrom (ARAÚJO, 1995; SILVA e MEDEIROS FILHO, 2006).

Esse fruto pode constituir fonte considerável de nutrientes na alimentação de populações regionais, pois apresenta teor elevado de lipídios, que varia de 27 a $35 \mathrm{~g} .100 \mathrm{~g}^{-1}$. Em decorrência disso, constitui boa fonte energética, variando de 250 a $350 \mathrm{kcal} .100 \mathrm{~g}^{-1}$ de polpa (CORDEIRO et al., 2013; LIMA et al., 2007). O perfil de ácidos graxos da polpa de pequi é favorável à saúde, sendo composto por $60 \%$ de ácidos graxos insaturados, com predominância do ácido oleico (BARRA et al., 2013). Destaca-se, ainda, que sua polpa pode ser considerada fonte potencial de antioxidantes naturais (MORAIS et al., 2013). Em relação ao perfil de minerais, a polpa de pequi possui teores consideráveis de magnésio, zinco e fósforo (OLIVEIRA et al., 2010; RAMOS e SOUZA, 2011).

O amplo período de oferta dos frutos de pequi e a diversidade de suas características físicas e químicas, já constatadas em frutos oriundos do estado de Goiás
(MOURA et al., 2013a, 2013b; VERA et al., 2007), sugerem que existam diferenças entre essas características em frutos procedentes de diferentes ambientes do Cerrado. Nesse sentido, o objetivo deste trabalho foi analisar as características físicas e nutricionais de frutos de pequizeiro oriundos dos estados de Tocantins, Goiás e Minas Gerais.

\section{Material e métodos}

Os frutos de pequi foram adquiridos na Central de Abastecimento de Goiás (Ceasa-GO), provenientes de três estados localizados no bioma Cerrado (30 kg/estado): Tocantins, TO (Nova Rosalândia, Pium, São Felix), em outubro de 2009; Goiás, GO (Faina, Mambaí e Santa Tereza), em dezembro de 2009; e Minas Gerais, MG (Campo Azul, Japonvar e Monte Alegre), em janeiro de 2010. O clima predominante nas diferentes regiões de procedência dos frutos, nos três estados, é o tropical semiúmido, com inverno seco. As condições ambientais nessas regiões foram similares no período de coleta dos frutos, exceto a menor precipitação total observada na região norte de Minas Gerais (Tabela 1).

Foram selecionados 30 frutos/estado em perfeitas condições morfológicas e no ponto ótimo de maduração, os quais foram submetidos às análises físicas e químicas. O processamento dos frutos compreendeu as seguintes etapas: higienização em água corrente, descascamento, despolpa, trituração e homogeneização da polpa em processador de alimentos (Walita, HL-3253), armazenamento em embalagem opaca $\mathrm{a}-18^{\circ} \mathrm{C}$, secagem dos caroços em estufa a $60^{\circ} \mathrm{C}$ por 30 horas (LIMA et al., 2007) e extração das sementes (amêndoa), com auxílio de equipamento tipo guilhotina.

A caracterização física do fruto e do caroço compreendeu a mensuração das seguintes variáveis: altura, diâmetro maior e menor do fruto $(\mathrm{mm})$; altura, diâmetro maior e menor dos caroços (mm); massa dos frutos, do mesocarpo, da polpa e das sementes (g); e número de caroços por fruto, conforme descrito por Vera et al. (2005). Todas as mensurações foram realizadas

Tabela 1. Condições ambientais nos municípios próximos às regiões de procedência dos frutos, nos meses de coleta, segundo informações do INMET.

\begin{tabular}{|c|c|c|c|c|c|}
\hline \multirow[t]{2}{*}{ Estado/Período } & \multirow[t]{2}{*}{ Estações INMET'1 } & \multirow{2}{*}{$\begin{array}{l}\text { Precipitação total } \\
\text { (mm) }\end{array}$} & \multicolumn{2}{|c|}{ Temperatura $\left({ }^{\circ} \mathbf{C}\right)$} & \multirow{2}{*}{$\begin{array}{c}\text { Umidade relativa } \\
\qquad(\%)\end{array}$} \\
\hline & & & Mínima & Máxima & \\
\hline $\begin{array}{l}\text { TO } \\
\text { Out/2009 }\end{array}$ & Palmas & 213,7 & 22,8 & 32,8 & 76,8 \\
\hline GO & Pirenópolis & 337,7 & 19,7 & 28,9 & 83,4 \\
\hline Dez/2009 & Posse & 325,2 & 19,8 & 29,2 & 75,9 \\
\hline MG & Pirapora & 77,9 & 21,2 & 32,7 & 72,8 \\
\hline Jan/2010 & Uberaba & 411,2 & 20,2 & 30,5 & 76,2 \\
\hline
\end{tabular}

${ }^{1}$ Estações INMET representativas dos municípios de coleta dos frutos: Palmas (Nova Rosalândia, Pium, São Felix); Pirenopólis (Faina); Posse (Mambaí e Santa Tereza); Pirapora (Campo Azul e Japonvar) e Uberaba (Monte Alegre); Fonte: Instituto Nacional de Meteorologia (INMET, 2014). 
com auxílio de balança semianalítica (Gehaka, BG8000) e paquímetro digital (Mitutoyo, 500-144B).

A caracterização química da polpa de pequi compreendeu a determinação da composição centesimal e a análise de minerais. A composição centesimal foi determinada em triplicata, por meio das análises de umidade (IAL, 2005), nitrogênio total (HORWITZ, 2002) e sua conversão em proteína, bruta utilizando-se o fator 6,25 (FAO, 1970), lipídios totais (BLIGH e DYER, 1959) e cinzas (HORWITZ, 2002). O teor de carboidratos totais foi estimado por diferença, subtraindo-se de 100 os valores obtidos para umidade, proteínas, lipídios e cinzas. A composição centesimal permitiu estimar o valor energético das amostras, considerando-se os fatores de conversão de Atwater, de 4, 4 e 9 kcal.g ${ }^{-1}$ para proteína, carboidratos e lipídios, respectivamente (MERRIL e WATT, 1973).

As cinzas das polpas de pequi foram solubilizadas em ácido clorídrico concentrado. Os teores dos minerais $\mathrm{Ca}, \mathrm{Mg}$, Fe e Zn foram caracterizados e quantificados por meio de espectrometria de absorção atômica, e os conteúdos de $\mathrm{Na}$ e K, por meio de emissão atômica, utilizando-se espectrômetro Perkin Elmer, Analyst-200. Os teores de fósforo foram determinados por espectrofotometria de ultravioleta visível (HORWITZ, 2002).

As características físicas foram analisadas por estatística descritiva (medidas de tendência central e variabilidade dos dados) e os resultados das análises químicas foram submetidos à análise de variância e teste para comparação de médias (Tukey a 5\% de probabilidade). Os cálculos estatísticos foram efetuados com auxílio do Programa Statistica, versão 7.0.

\section{Resultados e discussão}

A massa dos frutos oriundos de TO e GO (Tabela 2) apresentou valores inferiores à dos frutos de MG e ao valor médio encontrado para a massa de frutos procedentes de oito regiões do Cerrado, de 180,89 g (MOURA et al., 2013a). Cordeiro et al. (2013), em estudo com frutos de pequi oriundos do estado de Mato Grosso, observaram massa média dos frutos de $154 \mathrm{~g}$, valor superior ao encontrado nesse estudo para os frutos de TO e GO, e inferior ao valor médio constatado para os frutos de MG.

A massa da polpa dos frutos apresentou grande variação entre as regiões analisadas, sendo que os frutos de MG apresentaram valores superiores aos encontrados para os frutos de TO e GO (Tabela 2). O valor médio obtido para os frutos das regiões estudadas foi de duas a três vezes maior que os valores médios constatados na literatura, entre 3,31 g e 3,98 g (VERA et al., 2007), o que denota grande variabilidade em relação à massa da polpa dos frutos de pequi. Destaca-se que a polpa do pequi é a parte do fruto mais valorizada comercialmente, sendo muito utilizada na alimentação humana, assim como na indústria, para a elaboração de conservas e produtos derivados (ARÉVALO-PINEDO et al., 2010). Embora os frutos de MG tenham apresentado maior massa de

Tabela 2. Características físicas de frutos de pequi oriundos dos estados de Tocantins (TO), Goiás (GO) e Minas Gerais (MG).

\begin{tabular}{|c|c|c|c|c|c|c|c|c|c|c|c|c|}
\hline \multirow{2}{*}{ Característica } & \multicolumn{4}{|c|}{ TO } & \multicolumn{4}{|c|}{ GO } & \multicolumn{4}{|c|}{ MG } \\
\hline & Mín ${ }^{(1)}$ & Máx ${ }^{(2)}$ & Média & $\mathrm{CV}^{(3)}$ & Mín & Máx & Média & CV & Mín & Máx & Média & CV \\
\hline \multicolumn{13}{|l|}{ Fruto } \\
\hline Massa total (g) & 77,5 & 217,2 & 138,7 & 28,4 & 59,2 & 269,9 & 120,9 & 45,9 & 76,7 & 437,0 & 191,4 & 50,4 \\
\hline Altura (mm) & 49,1 & 70,5 & 59,2 & 9,4 & 51,0 & 71,3 & 60,5 & 7,2 & 55,7 & 90,4 & 69,6 & 13,9 \\
\hline Diâmetro maior (mm) & 55,7 & 96,2 & 72,4 & 16,3 & 47,9 & 95,8 & 64,3 & 20,9 & 53,6 & 113,3 & 74,3 & 20,9 \\
\hline Diâmetro menor (mm) & 43,4 & 65,7 & 56,4 & 9,0 & 45,3 & 70,6 & 55,2 & 13,2 & 47,7 & 87,2 & 63,7 & 18,5 \\
\hline Massa mesocarpo (g) & 62,0 & 179,9 & 108,7 & 31,1 & 44,8 & 203,9 & 94,5 & 45,1 & 53,8 & 343,9 & 144,9 & 54,0 \\
\hline \multicolumn{13}{|l|}{ Caroço } \\
\hline Número & 1,0 & 3,0 & 1,3 & 49,6 & 1,0 & 3,0 & 1,5 & 45,5 & 1,0 & 3,0 & 1,3 & 45,5 \\
\hline Massa total (g) & 13,9 & 59,2 & 28,2 & 36,3 & 8,3 & 68,7 & 24,7 & 58,9 & 14,7 & 87,2 & 43,1 & 47,9 \\
\hline Altura (mm) & 33,5 & 47,0 & 39,7 & 8,2 & 27,9 & 47,9 & 39,1 & 11,2 & 47,5 & 54,1 & 47,7 & 10,9 \\
\hline Diâmetro maior (mm) & 23,7 & 33,8 & 29,2 & 7,6 & 19,4 & 35,1 & 27,0 & 13,0 & 34,0 & 39,8 & 35,5 & 9,5 \\
\hline Diâmetro menor (mm) & 21,8 & 32,8 & 27,2 & 8,9 & 18,5 & 34,7 & 26,4 & 12,8 & 31,6 & 38,6 & 34,6 & 11,8 \\
\hline \multicolumn{13}{|l|}{ Polpa } \\
\hline Massa total (g) & 0,1 & 17,4 & 6,9 & 54,1 & 1,8 & 21,8 & 8,4 & 55,9 & 3,5 & 31,4 & 14,3 & 51,6 \\
\hline Rendimento $(\%)^{(4)}$ & 0,1 & 47,3 & 24,7 & 35,2 & 21,7 & 49,0 & 34,9 & 20,7 & 16,9 & 52,5 & 33,3 & 28,3 \\
\hline \multicolumn{13}{|l|}{ Semente } \\
\hline Massa total (g) & 0,2 & 2,2 & 0,9 & 42,5 & 0,1 & 3,9 & 1,1 & 88,0 & 0,1 & 5,3 & 2,1 & 65,2 \\
\hline Rendimento (\%) & 1,4 & 7,0 & 3,5 & 43,6 & 0,1 & 16,0 & 4,5 & 67,1 & 0,1 & 9,8 & 4,8 & 45,9 \\
\hline
\end{tabular}

(1)Mín: valor mínimo; (2)Máx: valor máximo; ${ }^{(3)} \mathrm{CV}$ : coeficiente de variação; ${ }^{(4)}$ Rendimento: relação entre massa total da polpa ou semente e massa do fruto. 
polpa, o rendimento de polpa foi próximo ao dos frutos procedentes de GO. Esses resultados podem contribuir para a seleção de frutos com maior rendimento de polpa, agregando assim valor e potencializando o uso comercial e industrial desse fruto.

A massa das sementes dos frutos de MG foi superior à dos frutos oriundos de TO e GO. As sementes dos frutos de GO apresentaram menor massa do que a relatada por Vera et al. (2007), que variou de 2,67 g a 3,22 g, conforme a região de origem. Em relação ao rendimento das sementes, os frutos de MG e GO apresentaram valores similares (Tabela 2). A semente ou amêndoa do pequi ainda é pouco aproveitada na alimentação humana, principalmente pela dificuldade na extração do endocarpo espinhoso. Assim, esses achados colaboram para um maior aproveitamento dessa semente, do ponto de vista comercial e industrial, visando o consumo humano e animal, ou a extração de óleo.

Estudos mostram que o pequizeiro, por ser uma planta nativa e ainda não cultivada em escala comercial, apresenta grande variabilidade nas características físicas de seus frutos (MOURA et al., 2013a, 2013b). Além disso, diferentes condições edafoclimáticas das regiões de procedência dos frutos, como precipitação total e tipo de solo, podem explicar os elevados coeficientes de variação observados neste estudo (Tabela 2).

Os frutos apresentaram polpa com umidade acima de $50 \%$, sendo os valores de GO e MG próximos entre si e ao relatado para frutos de pequi oriundos do estado de Mato Grosso (45,7 a 50,6 g.100 g ${ }^{-1}$ ) (CORDEIRO et al., 2013). Os frutos do TO se destacaram pelo elevado valor de umidade em relação aos demais (Tabela 3). Vale acrescentar que a umidade elevada da polpa dos frutos pode estar relacionada com a umidade relativa alta nas regiões de procedência dos frutos (Tabela 1) durante o período de safra.

Como esperado para polpa de frutos, a polpa de pequi possui baixo teor de proteína (Tabela 3), variando de 3,73 g.100 $\mathrm{g}^{-1}$ a 4,09 g.100 $\mathrm{g}^{-1}$, com diferença significativa apenas para os frutos de TO $(p<0,05)$. Esses resultados são compatíveis com a literatura, que registra valores entre 3 e 6 g.100 g-1 (LIMA et al., 2007; VERA et al., 2007).

Para o teor de lipídios (Tabela 3), observou-se valores semelhantes entre os frutos de GO e MG, coerentes com os valores relatados na literatura, de 20 a 35 g. $100 \mathrm{~g}^{-1}$ (CORDEIRO et al., 2013; LIMA et al., 2007; OLIVEIRA et al., 2010). Entretanto, para os frutos de TO, o conteúdo de lipídios foi inferior ao dos demais estados e aos valores disponíveis na literatura. Esse resultado pode ser um parâmetro interessante para o comércio desse fruto e seu uso na indústria de alimentos (Tabela 3). Vale ressaltar que o óleo da polpa de pequi é considerado de excelente qualidade nutricional pelo predomínio de ácidos graxos insaturados (BARRA et al., 2013), que contribuem para a promoção e manutenção da saúde.

Considerando-se uma porção de três caroços de pequi, observa-se grande variação do valor energético entre as três regiões, sobretudo para os frutos de TO (23,82 kcal), cuja porção resulta em um valor energético, no mínimo, duas vezes menor do que o dos outros estados (GO: 49,40 kcal e MG: 101,95 kcal), em decorrência do seu elevado teor de umidade e baixo teor de lipídios. Destaca-se que o baixo teor lipídico e reduzido valor energético da polpa dos frutos de TO é favorável à saúde, ao contrário da elevada densidade energética $\left(319,28 \mathrm{kcal} 100 \mathrm{~g}^{-1}\right)$ relatada na literatura para a polpa de pequi - Caryocar coriaceum (RAMOS e SOUZA, 2011).

Em relação ao conteúdo de minerais (Tabela 4), a polpa de pequi do TO é rica em zinco, fósforo e magnésio, pois satisfaz, no mínimo, 30\% da Ingestão Dietética Recomendada (RDA - Recommended Dietary Allowance) de referência por $100 \mathrm{~g}$ de alimento, e é fonte de ferro e potássio, pois satisfaz, no mínimo, 15\% da RDA (BRASIL, 1998). Além disso, possui um teor de cálcio duas vezes maior do que os teores observados para a polpa dos frutos dos demais estados e também de frutos de pequi provenientes do Ceará - 43,05 mg. $100 \mathrm{~g}^{-1}$ (OLIVEIRA et al., 2010). O valor elevado de cálcio na polpa do pequi nativo do TO é compatível com valores registrados na literatura, de 101,99 mg. $100 \mathrm{~g}^{-1}$, para pequis (Caryocar coriaceum) oriundos do Maranhão e Piauí (RAMOS e SOUZA, 2011). Esse teor considerável de cálcio constatado na polpa de

Tabela 3. Composição centesimal e valor energético da polpa de pequis oriundos dos estados de Tocantins (TO), Goiás (GO) e Minas Gerais (MG).

\begin{tabular}{lccr} 
Composição centesimal ${ }^{(\mathbf{1})} \mathbf{( g . 1 0 0 ~ \mathbf { ~ g } ^ { - 1 } )}$ & TO & GO & MG \\
Umidade & $72,17 \pm 0,72^{\mathrm{a}}$ & $56,21 \pm 0,47^{\mathrm{b}}$ & $54,78 \pm 0,34^{\mathrm{c}}$ \\
Proteínas & $4,09 \pm 0,06^{\mathrm{a}}$ & $3,73 \pm 0,00^{\mathrm{b}}$ & $3,73 \pm 0,01^{\mathrm{b}}$ \\
Lipídios & $8,39 \pm 0,25^{\mathrm{b}}$ & $24,27 \pm 1,60^{\mathrm{a}}$ & $26,15 \pm 0,85^{\mathrm{a}}$ \\
Cinzas & $0,85 \pm 0,05^{\mathrm{a}}$ & $0,63 \pm 0,02^{\mathrm{b}}$ & $0,67 \pm 0,02^{\mathrm{b}}$ \\
Carboidratos totais & 14,50 & 15,16 & 14,67 \\
VET (kcal.100 g $\mathrm{g}^{-1}$ ) & 149,87 & 293,99 & 308,95 \\
\hline
\end{tabular}

(1)Valores constituem média \pm desvios padrão de três replicatas de cada região; médias com letras iguais na mesma linha não apresentam diferenças significativas pelo teste de Tukey a 5\% de probabilidade; VET: valor energético total. 
Tabela 4. Composição de minerais da polpa de pequis oriundos dos estados de Tocantins (TO), Goiás (GO) e Minas Gerais (MG).

$\begin{array}{cccc}\text { Minerais }^{(\mathbf{1})}\left(\mathbf{m g} \cdot \mathbf{1 0 0} \mathbf{~ g}^{-\mathbf{1}}\right) & \text { TO } & \text { GO } & \text { MG } \\ \text { Cálcio } & 107,01 \pm 0,68^{\mathrm{a}} & 51,70 \pm 1,26^{\mathrm{c}} & 58,83 \pm 2,06^{\mathrm{b}} \\ \text { \% RDA }{ }^{(2)} & 10,70 & 5,17 & 5,88 \\ \text { Ferro } & 1,26 \pm 0,11^{\mathrm{a}} & 1,01 \pm 0,32^{\mathrm{a}} & 1,40 \pm 0,59^{\mathrm{a}} \\ \text { \% RDA } & 15,75 & 12,62 & 17,50 \\ \text { Zinco } & 3,51 \pm 0,70^{\mathrm{a}} & 3,81 \pm 0,93^{\mathrm{a}} & 2,81 \pm 0,70^{\mathrm{a}} \\ \text { \% RDA } & 31,91 & 34,64 & 25,54 \\ \text { Sódio } & 5,76 \pm 0,39^{\mathrm{a}, \mathrm{b}} & 7,29 \pm 0,79^{\mathrm{a}} & 4,02 \pm 0,71^{\mathrm{b}} \\ \text { \% RDA } & 0,38 & 0,49 & 0,27 \\ \text { Fósforo } & 294,24 \pm 10,62^{\mathrm{a}} & 125,64 \pm 5,73^{\mathrm{b}} & 136,62 \pm 2,13^{\mathrm{b}} \\ \text { \% RDA } & 42,03 & 17,95 & 19,52 \\ \text { Magnésio } & 132,06 \pm 5,70^{\mathrm{a}} & 69,32 \pm 1,43^{\mathrm{c}} & 84,29 \pm 2,94^{\mathrm{b}} \\ \text { \% RDA } & 31,44 & 16,50 & 20,07 \\ \text { Potássio } & 996,59 \pm 54,93^{\mathrm{a}} & 426,81 \pm 44,41^{\mathrm{b}} & 469,57 \pm 8,59^{\mathrm{b}} \\ \end{array}$

(1)Valores constituem média \pm desvios padrão de três replicatas de cada região; médias com letras iguais na mesma linha não apresentam diferenças significativas pelo teste de Tukey a $5 \%$ de probabilidade; (2)Recommended Dietary Allowance (RDA) para adultos saudáveis (31 a 50 anos): Cálcio = 1.000 mg/dia; Ferro = 8 mg/dia; Zinco = 11 mg/dia; Sódio = 1.500 mg/dia; Fósforo = 700 mg/dia; Magnésio = 420 mg/dia; Potássio $=4.700 \mathrm{mg} / \mathrm{dia}(\mathrm{IOM}, 2006)$.

pequi não é encontrado em polpa de frutos em geral, como abacaxi (14,49 mg.100 g $\left.{ }^{-1}\right)$, mamão (11,13 mg.100 g $\left.{ }^{-1}\right)$ e graviola (14,19 mg.100 g ${ }^{-1}$ ) (ALMEIDA et al., 2009), o que confere mais um atributo nutricional a esse fruto.

A polpa dos frutos de GO pode ser considerada rica em zinco e fonte de fósforo e magnésio, pois satisfaz, no mínimo, 30\% e 15\% da RDA desses elementos, respectivamente (BRASIL, 1998). A polpa de pequi oriundo de MG, por sua vez, pode ser considerada fonte de ferro, zinco, fósforo e magnésio (Tabela 4). Comparando-se as três regiões analisadas, houve diferenças significativas $(p<0,05)$ somente para os teores de cálcio e magnésio (Tabela 3).

Os teores de zinco constatados neste trabalho foram superiores aos valores relatados por Ramos e Souza (2011), de 1,75 mg. $100 \mathrm{~g}^{-1}$, e por Oliveira et al. (2010), de $1,37 \mathrm{mg} .100 \mathrm{~g}^{-1}$. Na perspectiva da segurança alimentar e nutricional, é interessante destacar os elevados teores de zinco na polpa de pequis das três regiões, uma vez que a deficiência desse mineral é comum em populações de risco e pode comprometer o desenvolvimento físico e intelectual dos indivíduos (PEDRAZA et al., 2013).

\section{Conclusão}

Os pequis provenientes de MG são mais indicados para a utilização no comércio e na indústria de alimentos, pelo maior rendimento de polpa. A polpa do pequi oriundo do TO difere das demais pela maior umidade, menor teor de lipídios, menor densidade energética e maior conteúdo de cálcio, além de ser rica em zinco e de ser fonte de ferro. Esses resultados indicam que a região de origem influencia nas características físicas e químicas dos frutos. Por isso, há necessidade de caracterização da região de origem e das espécies de pequizeiro para a comercialização dos frutos, e uso da polpa de pequi como matéria-prima nutritiva em diferentes sistemas alimentares.

\section{Referências}

ALMEIDA, M. M. B.; SOUSA, P. H.; FONSECA, M. L.; MAGALHÃES, C. E. C.; LOPES, M. F. G.; LEMOS, T. L. G. Avaliação de Macro e Microminerais em Frutas Tropicais Cultivadas no Nordeste Brasileiro. Ciência e Tecnologia de Alimentos, Campinas, v. 29, n. 3, p. 581-586, 2009. http://dx.doi. org/10.1590/S0101-20612009000300020

ARAÚJO, F. D. A Review of Caryocar brasiliense (Cariocaraceae): an Economically Valuable Species of the Central Brazilian Cerrados. Economic Botany, New York, v. 49, n. 1, p. 40-48, 1995. http://dx.doi.org/10.1007/BF02862276

ARÉVALO-PINEDO, A.; MACIEL, V. B. V.; CARVALHO, K. M.; COELHO, A. F. S.; GIRALDO-ZUÑIGA, A. D.; ARÉVALO, Z. D. S.; ALVIM, T. C. Processamento e Estudo da Estabilidade de Pasta de Pequi (Caryocar brasiliense). Ciência e Tecnologia de Alimentos, Campinas, v. 30, n. 3, p. 664-668, 2010. http:// dx.doi.org/10.1590/S0101-20612010000300015

BARRA, P. M. C.; OLIVEIRA, M. A. L.; NERY-ENES, B.; CARDOSO, L. M.; CESÁRIO, C. C.; MOREIRA, A. V. B.; PINHEIRO-SANT'ANA, H. M.; PELUZIO, M. C. G. Simultaneous Analysis of Saturated and Unsaturated Fatty Acids Present in Pequi Fruits by Capillary Electrophoresis. Química Nova, São Paulo, v. 36, n. 9, p. 1430-1433, 2013. http://dx.doi.org/10.1590/ S0100-40422013000900025

BATLLE-BAYER, L.; BATJES, N. H.; BINDRABAN, P. S. Changes in Organic Carbon Stocks Upon Land Use Conversion in the Brazilian Cerrado: a Review. Agriculture, Ecosystems and 
Características físicas e nutricionais de pequis oriundos dos estados de Tocantins, Goiás e Minas Gerais ALVES, A. M. et al.

Environment, Amsterdam, v. 137, n. 1-2, p. 47-58, 2010. http:// dx.doi.org/10.1016/j.agee.2010.02.003

BLIGH, E. G.; DYER, W. J. A Rapid Method of Total Lipid Extraction and Purification. Canadian Journal of Biochemistry and Physiology, Ottawa, v. 37, n. 8, p. 911-917, 1959. http:// dx.doi.org/10.1139/059-099

BRASIL. Agência Nacional de Vigilância Sanitária. Portaria $n^{\circ} 27$, de 13 de janeiro de 1998. Aprova o Regulamento Técnico Sobre a Informação Nutricional Complementar. Diário Oficial da União, Poder Executivo, Brasília, DF, 16 jan. 1998. Disponível em: <http://portal.anvisa.gov.br/wps/wcm/connect /9180ca00474581008d31dd3fbc4c6735/PORTARIA_27_1998. pdf?MOD=AJPERES $>$. Acesso em: 02 set. 2013.

CORDEIRO, M. W. S.; CAVALIERI, A. L. F.; FERRI, P. H.; NAVES, M. M. V. Características Físicas, Composição Químico-nutricional e dos Óleos Essenciais da Polpa de Caryocar brasiliense Nativo do Estado de Mato Grosso. Revista Brasileira de Fruticultura, Jaboticabal, v. 35, n. 4, p. 1127-1139, 2013.

FOOD AND AGRICULTURE ORGANIZATION OF THE UNITED NATIONS - FAO. Amino-acid Content of Foods and Biological Data on Proteins. Rome: FAO, 1970.

HORWITZ, W. (Ed.). Official Methods of Analysis of the Association of Official Analytical Chemists International. 17th ed. Arlington: AOAC, 2002. 1115 p.

INSTITUTE OF MEDICINE - IOM. Dietary Reference Intakes Research Synthesis: Workshop Summary. Washington: The National Academies Press, 2006. 298 p.

INSTITUTO ADOLFO LUTZ - IAL. Normas analíticas do Instituto Adolfo Lutz: Métodos Físico-químicos para Análise de Alimentos. 4. ed. Brasília: Ministério da Saúde, 2005. 1018 p.

INSTITUTO NACIONAL DE METEOROLOGIA - INMET. Banco de Dados Meteorológicos para Ensino e Pesquisa. Brasília. Disponível em: <http://www.inmet.gov.br/portal/index. php?r=bdmep/bdmep >. Acesso em: 23 jun. 2014.

LIMA, A.; SILVA, A. M. O.; TRINDADE, R. A.; TORRES, R. P.; MANCINI-FILHO, J. Composição Química e Compostos Bioativos Presentes na Polpa e na Amêndoa de Pequi (Caryocar brasiliense, Camb.). Revista Brasileira de Fruticultura, Jaboticabal, v. 29, n. 3, p. 695-698, 2007.

MERRIL, A. L.; WATT, B. K. Energy Value of Foods: Basis and Derivation. Washington: United States Departament of Agriculture, 1973. 105 p.

MORAIS, M. L.; SILVA, A. C. R.; ARAÚJO, C. R. R.; ESTEVES, E. A.; DESSIMONI-PINTO, N. A. V. Determinação do Potencial Antioxidante in vitro de Frutos do Cerrado Brasileiro. Revista
Brasileira de Fruticultura, Jaboticabal, v. 35, n. 2, p. 355-360, 2013.

MOURA, N. F.; CHAVES, L. J.; NAVES, R. V. Caracterização Física de Frutos de Pequizeiro (Caryocar brasiliense Camb.) do Cerrado. Revista Árvore, Viçosa, v. 37, n. 5, p. 905-912, $2013 a$. MOURA, N. F.; CHAVES, L. J.; NAVES, R. V.; AGUIAR, A. V.; SOBIERAJSKI, G. R. Variabilidade entre Procedências e Progênies de Pequizero (Caryocar brasiliense Camb.). Scientia Forestalis, Piracicaba, v. 41, n. 97, p. 103-112, 2013b.

NOVAES, P.; MOLINILLO, J. M. G.; VARELA, R. M.; MACÍAS, F. A. Ecological Phytochemistry of Cerrado (Brazilian Savanna) Plants. Phytochemistry Reviews, Netherlands, v. 12, n. 4, p. 839-855, 2013. http://dx.doi.org/10.1007/s11101-013-9315-3

OLIVEIRA, M. E. B.; GUERRA, N. B.; MAIA, A. H. N.; ALVES, R. E.; MATOS, N. M. S.; SAMPAIO, F. G. M.; LOPES, M. M. T. Características Químicas e Físico-químicas de Pequis da Chapada do Araripe, Ceará. Revista Brasileira de Fruticultura, Jaboticabal, v. 32, n. 1, p. 114-125, 2010.

RAMOS, K. M. C.; SOUZA, V. A. B. Características Físicas e Químico-nutricionais de Frutos de Pequizeiro (Caryocar coriaceum wittm.) em Populações Naturais da Região Meio-norte do Brasil. Revista Brasileira de Fruticultura, Jaboticabal, v. 33, n. 2, p. 500-508, 2011.

SANTANA, J. G.; NAVES, R. V. Caracterização de Ambientes de Cerrado com Alta Densidade de Pequizeiros (Caryocar brasiliense Camb.) na Região Sudeste do Estado de Goiás. Pesquisa Agropecuária Tropical, Goiânia, v. 33, n. 1, p. 1-10, 2003.

SILVA, M. A. P.; MEDEIROS FILHO, S. Morfologia de Fruto, Semente e Plântula de Piqui (Caryocar coriaceum Wittm.). Revista Ciência Agronômica, Fortaleza, v. 37, n. 3, p. 320-325, 2006.

PEDRAZA, D. F.; ROCHA, A. C. D.; SALES, M. C. Deficiência de Micronutrientes e Crescimento Linear: Revisão Sistemática de Estudos Observacionais. Ciência \& Saúde Coletiva, Rio de Janeiro, v. 18, n. 11, p. 3333-3347, 2013.

VERA, R.; NAVES, R. V.; NASCIMENTO, J. L.; CHAVES, L. J.; LEANDRO, W. M.; SOUZA, E. R. B. Caracterização Física de Frutos do Pequizeiro (Caryocar brasiliense Camb.) no Estado de Goiás. Pesquisa Agropecuária Tropical, Goiânia, v. 35, n. 2, p. 71-79, 2005.

VERA, R.; SOUZA, E. R. B.; FERNANDES, E. P.; NAVES, R. V.; SOARES JÚNIOR, M. S.; CALIARI, M.; XIMENES, P. A. Caracterização Física e Química de Frutos do Pequizeiro (Caryocar brasiliense Camb.) Oriundos de Duas Regiões no Estado de Goiás, Brasil. Pesquisa Agropecuária Tropical, Goiânia, v. 37, n. 2, p. 93-99, 2007. 